$\mathrm{p}=0.03$ ), and to both IcSSc (OR $2.80,95 \% \mathrm{Cl} 1.16$ to $6.84, \mathrm{p}=0.02)$ and dcSSc (OR $3.42,95 \% \mathrm{Cl} 1.20$ to $9.72, \mathrm{p}=0.02$ ) subtypes. The $S R p 55$ rs2235611 A minor allele and AA genotype showed a significant risk association with susceptibility to SSc-related pulmonary fibrosis (A allele: OR $1.39,95 \% \mathrm{Cl} 1.00$ to $1.93, p=0.046$; AA genotype: $\mathrm{OR} 3.95,95 \% \mathrm{Cl} 1.48$ to $10.54, \mathrm{p}=0.006)$. A trend toward an association between the AA genotype and anti-Scl70 antibody-positive SSc was also found (OR 2.82, $95 \% \mathrm{Cl} 0.95$ to $8.37, \mathrm{p}=0.06$ ). Both rs2235611 A allele and $\mathrm{AA}$ genotype were significantly associated with the SSc subset without digital ulcers (A allele: OR 1.33, 95\% Cl 1.01 to $1.75, p=0.04$; AA genotype: OR $3.26,95 \% \mathrm{Cl}$ 1.32 to $8.03, p=0.01$ ).

Conclusion: The SRp55 rs2235611 polymorphism is associated with susceptibility to SSc and, in particular, with SSc-related pulmonary fibrosis and peripheral vascular phenotype, consistent with a role of VEGF-A pre-mRNA alternative splicing in the development of pulmonary fibrosis and impairment of angiogenesis. Further replication studies are warranted to confirm our findings in independent SSc cohorts.

Disclosure of Interests: Eloisa Romano: None declared, Mirko Manetti: None declared, Joanna Kosalka-Wegiel: None declared, Bianca Saveria Fioretto: None declared, Irene Rosa: None declared, Elena Sticchi: None declared, Serena Guiducci: None declared, Silvia Bellando-Randone: None declared, Lidia Ibba-Manneschi: None declared, Marco Matucci-Cerinic Grant/research support from: Actelion, MSD, Bristol-Myers Squibb, Speakers bureau: Acetelion, Lilly, Boehringer Ingelheim

DOI: 10.1136/annrheumdis-2020-eular.1707

\section{SAT0283 SOLUBLE GUANYLATE CYCLASE REDUCED THE GASTROINTESTINAL FIBROSIS IN BLEOMYCIN- INDUCED MOUSE MODEL OF SYSTEMIC SCLEROSIS}

Y. Yamamoto ${ }^{1}$, T. Okano ${ }^{1}$, T. Nagamoto ${ }^{1}$, Y. Fujikawa ${ }^{1}$, Y. Ichise ${ }^{1}$, H. Yamada ${ }^{1}$, I. Naka ${ }^{1}$, Y. Ueda' ${ }^{1}$ K. Akashi', S. Sendo ${ }^{1}$, A. Onishi ${ }^{1}$, J. Saegusa', A. Morinobu ${ }^{1}$. ${ }^{1}$ Department of Rheumatology and Clinical Immunology, Kobe University Graduate School of Medicine, Kobe, Japan

Background: Systemic scleroderma (SSc) is a chronic autoimmune-mediated connective tissue disorder. Although the etiology of the disease remains undermined, SSc is characterized by fibrosis and proliferative vascular lesions of the skin and internal organs. SSc involves the gastrointestinal tract in more than $90 \%$ of patients ${ }^{1}$. Soluble guanylate cyclase (sGC) is used to treat pulmonary artery hypertension (PAH), and has been shown to inhibit experimental skin fibrosis ${ }^{2}$.

Objectives: The aim of this study is to investigate whether bleomycin (BLM)treated mice show gastrointestinal fibrosis, and find a therapeutic strategy to the lesion.

Methods: Female C57BL/6J mice were treated with BLM or normal saline by subcutaneous implantation of osmotic minipump. These mice were sacrificed on day 28 or day 42 . Gastrointestinal pathologies were examined by Masson Trichrome staining. The expression of fibrosis-related genes in gastrointestinal tract were analyzed by real-time PCR, and the levels of collagen in the tissue was measured by Sircol collagen assay. To evaluate peristaltic movement, the small intestinal transport (ITR\%) was calculated as [Dyeing distancex(Duodenum- Appendix)] $-1 \times 100(\%)$. We treated BLM-treated mice with soluble guanylate cyclase (sGC) or DMSO orally and analyzed them on day 42.

Results: Histological examination revealed that fibrosis from lamina propria to muscularis mucosa in the esophagus was significantly increased in BLMtreated mice, suggesting that BLM induces esophageal fibrosis in C57BL/6J mice. In addition, the levels of Col3a1 and CTGF were significantly increased in BLM-treated mice. More severe fibrosis was observed in the mice sacrificed on day 42 than the mice sacrificed on day 28 . The ITR\% was found to be significantly lower in BLM-treated mice, suggesting that gastrointestinal peristaltic movement was reduced in BLM-treated mice. Furthermore, we demonstrated that SGC treatment significantly reduced fibrosis of esophagus and intestine in BLM-treated mice, by histological examination and Sircol collagen assay.

Esophagus (Masson's trichrome stain $\times 100$ )
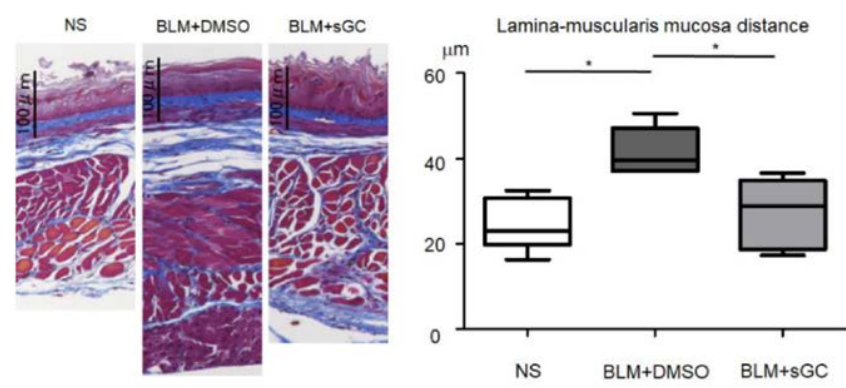

* One way ANOVA Newman-Keuls

Conclusion: These findings suggest that BLM induces gastrointestinal fibrosis in C57BL/6J mice, and treatment with SGC improves the BLM-induced gastrointestinal lesion.

\section{References:}

[1] Anton Emmanuel. Current management of the gastrointestinal complications of systemic sclerosis. Nat Rev Gastroenterol Hepatol. 2016; 13: 461-472.

[2] Clara Dees, et al. Stimulators of Soluble Guanylate Cyclase (sGC) Inhibit experimental Skin Fibrosis of Different Aetiologies. Ann Rheum Dis. 2015;74 (8): 1621-5

Acknowledgments: : None.

Disclosure of Interests: None declared

DOI: 10.1136/annrheumdis-2020-eular.2768

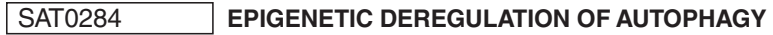 PROMOTES FIBROSIS IN SYSTEMIC SCLEROSIS}

A. Zehender ${ }^{1}$, N. Y. Lin ${ }^{1}$, Y. N. LI ${ }^{1}$, A. H. Györfi ${ }^{1}$, C. Bergmann ${ }^{1}$, A. Ramming ${ }^{1}$,

G. Schett2, J. Distler2. ' University Erlangen-Nürnberg (FAU) and University Hospital Erlangen, Department of Internal Medicine 3 - Rheumatology and Immunology, Erlangen, Germany; ${ }^{1}$ University Erlangen-Nürnberg (FAU) and University Hospital Erlangen, Department of Internal Medicine 3 Rheumatology and Immunology, Erlangen, Germany

Background: Autophagy is catabolic process allowing cells to degrade unnecessary or dysfunctional cellular organelles. Failure of appropriate regulation of autophagy, however, can severely perturb tissue homeostasis. Several stimuli present in fibrosis such as pro-fibrotic cytokines are known to activate autophagy. Objectives: The objective of this work was to characterize the regulation of autophagy in systemic sclerosis (SSc) and to decipher its role in the pathogenesis of SSc.

Methods: Activation of autophagy in SSc skin and matched tissue samples from healthy individuals was assessed by immunofluorescence staining for ATG7, BECLIN1 and P62. We generated $A \operatorname{tg} 7^{\mathrm{fl} / \mathrm{fl}} \times \mathrm{Col}$ 1 a2; CreER mice to selectively disable autophagy in fibroblasts. The role of the autophagy was investigated in the model of bleomycin- and T $\beta$ Rlact-induced dermal and pulmonary fibrosis. Overexpression of Myst1 was achieved by adenovirus encoding for Myst1. Collagen release and protein expression were measure by Western blot. Target genes were analyzed by RT-PCR. Co-immunoprecipitation and reporter assay were performed to study physical and functional interactions between MYST1 and SMAD3. To monitor the autophagic flux in vitro and in vivo we generated adenoviral vectors encoding for tandem fluorescent-tagged LC3 (mRFP-EGFP-LC3).

Results: Transforming growth factor- $\beta$ (TGF $\beta$ ) activates autophagy by an epigenetic mechanism to amplify its profibrotic effects. TGF $\beta$ induces autophagy in fibrotic diseases by SMAD3-dependent downregulation of the H4K16 histone acetyltransferase MYST1, which regulates the expression of core components of the autophagy machinery such as ATG7 and BECLIN1. Activation of autophagy in fibroblasts promotes collagen release and is both, sufficient and required, to induce tissue fibrosis. Forced expression of MYST1 abrogates the stimulatory effects of TGF $\beta$ on autophagy and re-establishes the epigenetic control of autophagy in fibrotic conditions. Interference with the aberrant activation of autophagy inhibits TGF $\beta$-induced fibroblast activation and ameliorates experimental dermal and pulmonary fibrosis. These findings link uncontrolled TGF $\beta$ signaling to aberrant autophagy, deregulated epigenetics in fibrotic diseases and may open new avenues for therapeutic intervention in fibrotic diseases.

Conclusion: We demonstrate that the epigenetic control of autophagy is disturbed by a TGF $\beta$-dependent downregulation of the H4K16 histone acetyltransferase MYST1. The increased activation of autophagy induces fibroblast-to-myofibroblast transition and promotes fibrotic tissue remodeling Re-expression of MYST1 prevents aberrant autophagy, limits the profibrotic effects of TGF $\beta$ and ameliorates experimental fibrosis. Restoration of the 
epigenetic control of autophagy might thus be a novel approach to ameliorate fibrotic tissue remodeling.

References:

[1] Wynn, T.A. Cellular and molecular mechanisms of fibrosis. J Pathol 214, 199210 (2008).

[2] Distler, J.H., et al. Review: Frontiers of Antifibrotic Therapy in Systemic Sclerosis. Arthritis \& rheumatology (Hoboken, N.J.) 69, 257-267 (2017).

[3] Gyorfi, A.H., Matei, A.E. \& Distler, J.H.W. Targeting TGF-beta signaling for the treatment of fibrosis. Matrix biology: journal of the International Society for Matrix Biology 68-69, 8-27 (2018).

[4] Wang, C.W. \& Klionsky, D.J. The molecular mechanism of autophagy. Mol Med 9, 65-76 (2003)

[5] Hernandez-Gea, V., et al. Autophagy releases lipid that promotes fibrogenesis by activated hepatic stellate cells in mice and in human tissues. Gastroenterology 142, 938-946 (2012)

Disclosure of Interests: Ariella Zehender: None declared, Neng Yu Lin: None declared, Yi-Nan Li: None declared, Andrea-Hermina Györfi: None declared, Christina Bergmann: None declared, Andreas Ramming Grant/research support from: Pfizer, Novartis, Consultant of: Boehringer Ingelheim, Novartis, Gilead, Pfizer, Speakers bureau: Boehringer Ingelheim, Roche, Janssen, Georg Schett Speakers bureau: AbbVie, BMS, Celgene, Janssen, Eli Lilly, Novartis, Roche and UCB, Jörg Distler Grant/research support from: Boehringer Ingelheim, Consultant of: Boehringer Ingelheim, Paid instructor for: Boehringer Ingelheim, Speakers bureau: Boehringer Ingelheim

DOI: 10.1136/annrheumdis-2020-eular.3503

\section{SAT0285 VISUALISATION OF THE ACTIVE CALCIFICATION PROCESS WITH 18-F SODIUM FLUORIDE PET/CT IN LIMITED CUTANEOUS SYSTEMIC SCLEROSIS WITH CALCINOSIS CUTIS IS FEASIBLE: A PILOT STUDY}

I. M. Atzeni ${ }^{1}$, E. M. Hogervorst ${ }^{1}$, G. M. Swart ${ }^{1}$, K. De Leeuw ${ }^{2}$, M. Bij $\left.\right|^{3}$, R. Bos ${ }^{4}$, J. Westra ${ }^{2}$, G. Diercks ${ }^{5}$, H. Van Goor ${ }^{5}$, M. C. Bolling ${ }^{6}$, R. Slart ${ }^{7}$, D. J. Mulder ${ }^{1} .{ }^{1}$ University of Groningen, University Medical Center Groningen, Department of Internal Medicine, Division Vascular Medicine, Groningen, Netherlands; ${ }^{2}$ University of Groningen, University Medical Center Groningen, Department of Rheumatology \& Clinical Immunology, Groningen, Netherlands; ${ }^{3}$ Martini Hospital, Department of Rheumatology, Groningen, Netherlands; ${ }^{4}$ Medical Center of Leeuwarden, Department of Rheumatology, Leeuwarden, Netherlands; ${ }^{5}$ University of Groningen, University Medical Center Groningen, Department of Pathology \& Medical Biology, Groningen, Netherlands; ${ }^{6}$ University of Groningen, University Medical Center Groningen, Department of Dermatology, Groningen, Netherlands; ${ }^{7}$ University of Groningen, University Medical Center Groningen, Department of Nuclear Medicine \& Molecular Imaging, Groningen, Netherlands

Background: Calcinosis cutis is a major daily challenge to patients with longstanding systemic sclerosis (SSc), negatively affecting their quality of life. Unfortunately, treatment options are very limited due to lack of understanding of the pathogenetic process. Currently, calcinosis cutis is only detected at its irreversible end-stage. Early detection of calcinosis cutis could putatively allow early disease-modifying interventions and monitor treatment effects.

Objectives: The aim of the current study is to assess the feasibility of visualising "active" micro-calcifications with 18-F Sodium Fluoride (NaF) PET scanning, compared to low-dose CT in patients with clinically overt calcinosis cutis.

Methods: This was a cross-sectional, observational, pilot study. All patients met 2013 ACR/EULAR criteria for SSc. Patients underwent a whole body NaF PET/ low-dose CT scan, scanned 90 minutes post-injection. (Sub)cutaneous calcifications were described and assessed on $\mathrm{NaF}$ PET, which was compared to CT images by two independent investigators.

Results: A total of 10 female patients with limited cutaneous SSc [median age 56 years (IQR 52-66), median disease duration 17 years (8-19), PAH 10\%, ILD $20 \%$ ] were included, and compared to 10 controls [70 years (65-73)]. NaF uptake showed normal distribution throughout the skeletal bones, arterial tree, and visceral organs, which was comparable between patients and controls. Additionally, $\mathrm{NaF}$ uptake was visible in the skin of all SSc patients, but in none of the controls. Cutaneous NaF uptake largely correlated with clinical calcifications. Most common sites of cutaneous $\mathrm{NaF}$ uptake were fingers (6 patients) and knees (7 patients). Only $5 \%$ of the NaF positive lesions were not accompanied by visible calcifications on CT. Furthermore, of all calcified lesions seen on CT, $51 \%$ showed uptake on $\mathrm{NaF}$ PET. Small lesions $(<1 \mathrm{~cm})$, were generally only visible on $\mathrm{CT}$, due to lower resolution of $\mathrm{NaF}$ PET.

Conclusion: Imaging of "active" calcinosis cutis in limited cutaneous systemic sclerosis is feasible using NaF PET scanning. Most clinically overt calcifications and half of those seen on CT were positive for $\mathrm{NaF}$ uptake. Whether these "active" calcifications behave differently in terms of faster progression, clinical complaints, and infection risk, and whether these are potentially suitable for disease modifying interventions is subject to future study.

Fig. 1 Clinical calcinosis cutis with corresponding images of 18F-NaF PET/CT-scan

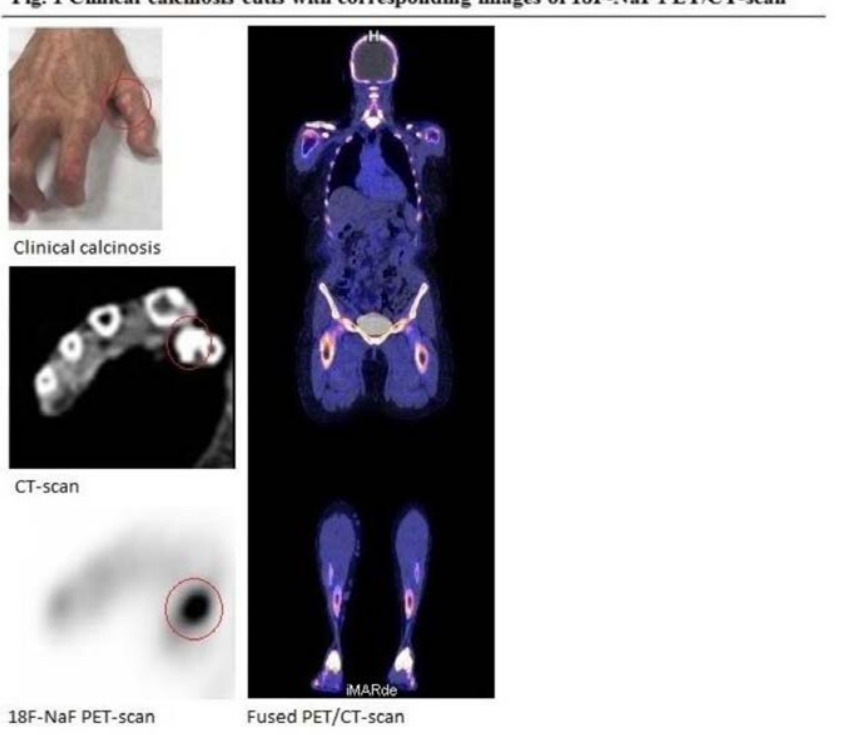

Disclosure of Interests: None declared DOI: 10.1136/annrheumdis-2020-eular.4629

\section{SAT0286 BIOLOGICAL CORRELATES OF RADIOGRAPHIC FEATURES OF INTERSTITIAL LUNG DISEASE IN SYSTEMIC SCLEROSIS: AN IN DEPTH ANALYSIS OF BRONCHOALVEOLAR PROTEINS OF SCLERODERMA LUNG STUDY I PARTICIPANTS}

E. Volkmann ${ }^{1}$, D. Tashkin ${ }^{1}$, N. Li ${ }^{1}$, G. Kim ${ }^{1}$, J. Goldin ${ }^{1}$, A. Harui ${ }^{1}$, M. Roth ${ }^{1}$.

${ }^{1}$ UCLA, Los Angeles, United States of America

Background: Systemic sclerosis-related interstitial lung disease (SSc-ILD) involves a combination of inflammation, fibrosis and vascular pathology that is typically assessed on CT imaging as a mixture of ground-glass opacification (GGO) and fibrotic changes. We hypothesized that proteins recovered from bronchoalveolar lavage (BAL) could be used to probe the underlying pathobiology associated with GGO and fibrotic changes.

Objectives: (1) To assess the relationship between 68 unique BAL proteins measured in participants of Scleroderma Lung Study (SLS) $I^{1}$ and radiographic and physiologic measures of ILD; (2) To identify inter-correlations among specific proteins to enlighten our understanding of how specific biological pathways contribute to SSc-ILD.

Methods: Bronchoscopy was performed on 144 of the 158 participants in SLS I with 103 BAL samples available for analysis. BAL was lyophilized, concentrated 10X and used in a multiplex protein analysis for 68 different cytokines chemokines and other factors. Kendall tau correlations were performed to assess the relationship between individual proteins and baseline measures of pulmonary function and quantitative CT scores for fibrosis, GGO and total ILD. Those proteins found to correlate significantly with at least 2 clinical measures of ILD were entered into a cluster analysis with inter-correlations expressed as a heatmap.

Results: Significant correlations were observed between fibrosis scores and several biologic pathways including pro-fibrotic factors (transforming growth factor beta [TGF- $\beta$ ], platelet-derived growth factor [PDGF]), proteins involved in tissue remodeling (Matrix metallopeptidase [MMP]-1,7,8,9; Hepatocyte growth factor [HGF]), and those involved in monocyte/macrophage migration and activation (Monocyte chemoattractant protein [MCP]-1,3; macrophage colony-stimulating factor [MCSF]). These same pathways correlated with the diffusing capacity for carbon monoxide (DLCO). In contrast, GGO scores correlated primarily with immune and inflammatory mediators (interleukin [IL]-5,8,13,15, IL-1 receptor antagonist and interferon gamma) with only limited overlap to proteins that related to fibrosis. Vascular endothelial growth factor (VEGF) levels were lower in patients with more extensive GGO, fibrosis and diffusion impairment, suggesting that vascular changes are a central feature of SSc-ILD. Specific proteins were highly correlated with one another in a pattern suggesting biologically-related networks (Figure) that might provide additional insight regarding disease pathogenesis. 